\title{
The link between Toxoplasma gondii infections and higher mortality in COVID-19 patients having schizophrenia
}

\author{
Kevin $\operatorname{Roe}^{1}$ (D)
}

Received: 14 September 2021 / Accepted: 27 September 2021 / Published online: 4 October 2021

(c) The Author(s), under exclusive licence to Springer-Verlag GmbH Germany 2021

\begin{abstract}
A strong link between schizophrenia and a higher mortality rate from SARS-CoV-2 infections has been reported for schizophrenia patients, with a mortality odds ratio (OR) of 2.67 compared to normal patients, after adjustment of the OR for age, sex, race and extra risk factors. In addition, an extensive number of papers have reported a very strong link between schizophrenia and Toxoplasma gondii infections. A meta-analysis of 38 studies of links between schizophrenia and T. gondii antibody seroprevalence resulting from previous infections indicated that the likelihood of $T$. gondii infection in schizophrenia patients was 2.7 times higher than the general population. In other words, the meta-analysis indicated that schizophrenia patients had an odds ratio of 2.7 of $T$. gondii infection compared to the general population. This indicates that compared to the general population, schizophrenia patients have virtually the same odds ratio for having a $T$. gondii infection and for mortality from a COVID-19 infection. This suggests that $T$. gondii infections, directly or indirectly, have a relationship with higher mortality in COVID-19 patients having schizophrenia. This conclusion would also apply to the general population.
\end{abstract}

Keywords Brain infections $\cdot$ Latent infections $\cdot$ Protozoa $\cdot$ Protozoan infections $\cdot$ COVID-19 mortality

A strong link between schizophrenia and a higher mortality rate from SARS-CoV-2 infections has been reported for schizophrenia patients, with a mortality odds ratio (OR) of 2.67 compared to normal patients, after adjustment of the OR for age, sex, race and extra risk factors [1]. In addition, an extensive number of papers have reported a very strong link between schizophrenia and $T$. gondii infections [2].

This is interesting, because a meta-analysis of 38 studies of links between schizophrenia and T. gondii antibody seroprevalence resulting from previous infections indicated that the likelihood of $T$. gondii infection in schizophrenia patients was 2.7 times higher than the general population [2]. In other words, the meta-analysis indicated that schizophrenia patients had an odds ratio of 2.7 of $T$. gondii infection compared to the general population [2].

This match is very interesting, because this indicates that compared to the general population, schizophrenia patients have virtually the same odds ratio for having a $T$. gondii infection and for mortality from a COVID-19 infection. This

Kevin Roe

kevin.roe@att.net

1 San Jose, CA, USA suggests that $T$. gondii infections, directly or indirectly, have a relationship with higher mortality in COVID-19 patients having schizophrenia. This conclusion can also apply to the general population. One question is how these two pathogen infections, a protozoan parasite infection and a viral infection, could be linked together to cause a higher COVID-19 mortality? In other words, what is the common factor that could connect these totally different infections?

It has been hypothesized that a SARS-CoV-2 virus infection can act together with $T$. gondii infections, through mutually beneficial induced immune cell dysfunctions, such as T-cell exhaustion [3]. This is because one latent pathogen infection, the protozoan parasite $T$. gondii that infects the brain and muscles, can create T-cell exhaustion [3]. In addition, T-cell exhaustion, particularly CD8 T-cell exhaustion, is also seen in COVID-19 patients with more severe outcomes $[4,5]$.

There is arguably substantial direct evidence, including activated microglia and microglial nodules, of $T$. gondii infections that were observed in the brain autopsies of 41 individuals who died from COVID-19 [3]. Furthermore, a match in the odds ratios between the COVID-19 mortality rates and the odds ratio of $T$. gondii infections has been observed for various categories of people, based on age, 
gender and body mass index [3]. In addition, a considerable match has also been observed between the symptoms of COVID-19 and the symptoms of active T. gondii infections [3].

Not all $T$. gondii infections have the same consequences. It has been observed that $T$. gondii infections can differ in causing T-cell exhaustion, because $T$. gondii has a large number of different genetic types with vast differences in their immunological effects; and it has been noted that T-cell exhaustion may or may not be present because of certain drug treatments previously given to patients during their active $T$. gondii infections [3].

In conclusion, $T$. gondii infections have an extensively documented involvement in several schizophrenia cases. A statistically increased mortality rate for COVID-19 patients having schizophrenia can be logically explained by a subset of schizophrenia patients having latent $T$. gondii infections that cause immune dysfunctions, such as $\mathrm{CD} 8 \mathrm{~T}$ cell exhaustion. This could cause more severe outcomes for these COVID-19 patients. In addition, the matches in corresponding patient categories between the odds ratios of COVID-19 mortality and the odds ratios of $T$. gondii infection suggest that certain genetic types of $T$. gondii, especially in cases without early protozoan parasite drug treatments, can induce immune dysfunctions, such as T-cell exhaustion, which can cause more severe outcomes for several COVID-19 patient categories.

Funding This research did not receive any specific grant from funding agencies in the public, commercial, or not-for-profit sectors.
Data availability Data sharing is not applicable to this article as no new data were created or analyzed in this study.

\section{Declarations}

Conflict of interest The author has no potential conflicts of interest.

Ethical approval No ethical approval was required as this is an article with no original research data.

\section{References}

1. Nemani K, Li C, Olfson M, Blessing EM, Razavian N, Chen J, Petkova E, Goff DC (2021) Association of psychiatric disorders with mortality among patients with COVID-19. JAMA Psychiat. https://doi.org/10.1001/jamapsychiatry.2020.4442

2. McConkey GA, Martin HL, Bristow GC, Webster JP (2013) Toxoplasma gondii infection and behaviour-location, location, location? J Exp Biol 216(1):113-119

3. Roe K (2021) A role for T-cell exhaustion in long COVID-19 and in severe outcomes in several categories of COVID-19 patients. J Neurosci Res. https://doi.org/10.1002/jnr.24917

4. Zheng M, Gao Y, Wang G, Song G, Liu S, Sun D, Xu Y, Tian Z (2020) Functional exhaustion of antiviral lymphocytes in COVID19 patients. Cell Mol Immunol 17:533-535. https://doi.org/10. 1038/s41423-020-0402-2

5. Zheng HY, Zhang M, Yang CX, Zhang N, Wang XC, Yang XP, Dong XQ, Zheng YT (2020) Elevated exhaustion levels and reduced functional diversity of $\mathrm{T}$ cells in peripheral blood may predict severe progression in COVID-19 patients. Cell Mol Immunol 17:541-543. https://doi.org/10.1038/s41423-020-0401-3 